\title{
Non-Steroidal Anti-Inflammatory Drug Use and Recovery after Achilles Tendon Rupture
}

\author{
V. Franco ${ }^{1}$, J. Grotts ${ }^{2}$, J. C. Lin' ${ }^{3}$, M. K. Fong ${ }^{3}$, M. S. Vasquez ${ }^{3}$ \\ 1 Departments of Emergency Medicine and Orthopedic Surgery, University of California Los Angeles, Los \\ Angeles, CA \\ 2 University of California Los Angeles Medical Center, Los Angeles, Medical Center, Los Angeles, CA \\ 3 Kaiser Permanente Los Angeles Medical Center, Los Angeles, CA
}

\section{CORRESPONDING AUTHOR:}

Vanessa Franco

Departments of Emergency Medicine and Orthopedic Surgery

University of California Los Angeles

924 Westwood Blvd Suite 300

Los Angeles, CA 90095

Phone: 412-477-7412

E-mail:vfranco@mednet.ucla.edu

DOI:

10.32098/mltj.03.2019.02

LEVEL OF EVIDENCE: 3

\begin{abstract}
SUMMARY
Background. The effect of non-steroidal anti-inflammatory drugs (NSAIDs) on recovery after Achilles tendon rupture is unknown. This is the first study examining the relationship between NSAID use and recovery after Achilles tendon rupture in humans.

Methods. Adult patients presenting between 01/01/14 and 03/30/2018 with acute Achilles tendon rupture were asked to complete a survey in the Spring of 2018 to determine whether they took NSAIDs after rupture. Their recovery was quantified in three ways: 1) the Achilles Tendon Total Rupture Score (ATRS), 2) the duration of time to walk again normally, and 3) the incidence of tendon re-rupture. Recovery was compared across NSAID and non-NSAID users.

Results. Fifty-three percent of 361 patients who completed the survey used NSAIDs following tendon rupture. NSAID users reported a significantly lower ATRS (69) than non-NSAID users $(77 ; \mathrm{p}<0.005)$. There was no difference in time to walk again normally or incidence of re-rupture. After controlling for age, sex, operative versus non-operative treatment, and time from diagnosis to survey completion, NSAID use was still associated with a significantly lower ATRS $(p=0.003)$.

Conclusion. NSAID use was associated with a lower ATRS after Achilles tendon rupture.
\end{abstract}

\section{KEY WORDS}

ATRS; bealing; inflammation; musculoskeletal

\section{SUMMARY}

The initial management of Achilles tendon rupture often involves pain control, including the administration of non-steroidal anti-inflammatory drugs (NSAIDs). While there is some evidence that NSAIDs may impair bone healing (1-4), the effect of NSAIDs on tendon healing is less clear. In vitro, NSAID exposure after tendon injury was found to inhibit proliferation and migration of tendon cells, but increase collagen synthesis (5). A study in rat Achilles tendons demonstrated that ibuprofen upregulates matrix metalloproteinases, which are known to degrade collagen (6). Consistent with this finding, the early administration of NSAIDs after injury in rats was found to impede recovery of the Achilles tendon (7) and the patellar tendon $(8,9)$. In contrast, a separate study found that NSAID use after Achilles tendon transection in rats was associated with equivalent failure loads post-operatively (10). To complicate the issue, early NSAID administration after supraspinatus tendon injury in rats was found to impair recovery, but late NSAID administration was either beneficial or had no effect $(11,12)$.

The aforementioned studies have limited applicability, as most involved rodents undergoing surgical transection of the Achilles or other tendons, which do not accurately represent Achilles tendon rupture in humans (13). Furthermore, tendon recovery in most studies was assessed two weeks following transection. The long-term effects of NSAIDs on tendon healing have not been studied. Due to the lack of human subjects, the artificial nature of injury, and the limited time points of assessment, it remains unclear whether NSAID use following Achilles tendon rupture impacts functional recovery in humans. 
Given the frequency with which patients are administered NSAIDs following injuries, it is imperative to determine how this practice may impact a patient's recovery after Achilles tendon rupture. In an initial step towards understanding any relationship between NSAID use and recovery following Achilles tendon rupture, a survey was sent to all patients presenting with an Achilles tendon rupture to assess whether NSAID use after Achilles tendon rupture was associated with worse functional outcomes. We hypothesized that NSAID use would be associated with worse recovery, as indicated by a lower Achilles Tendon Total Rupture Score (ATRS).

\section{METHODS}

\section{Patient Population}

Institutional review board approval was obtained for this study and the study meets accepted ethical standards (14). All patients aged 18 and older presenting with an initial diagnosis of Achilles tendon rupture were identified by searching for initial encounters with the following ICD-9 or ICD-10 codes: 727.67 Rupture of Achilles tendon, 845.09 Achilles tendon tear, 891.2 Achilles tendon laceration, M66.871 Nontraumatic rupture of right or bilateral Achilles tendon, M66.872 Nontraumatic rupture of left or bilateral Achilles tendon, S86.011A Right Achilles tendon tear, S86.012A Left Achilles tendon tear, S86.021A Right Achilles tendon laceration, S86.022A Left Achilles tendon laceration, or S86.092A Laceration of unspecified Achilles tendon.

Patients who suffered an Achilles tendon rupture between January 1, 2014 and December 31, 2015 were sent invitations to participate in March of 2018. Patients suffering an Achilles tendon rupture between January 1, 2016 and March 30, 2018 were sent survey invitations in July of 2018. A non-parametric post-hoc power analysis computed a power of $82.7 \%$.

The survey was created using SurveyMonkey. Patients were given a unique de-identified code via email to maintain anonymity throughout the survey.

\section{Outcomes}

The Achilles Tendon Total Rupture Score (ATRS) was selected to be the primary outcome because it is a patient-reported externally validated score that quantifies functional outcome after Achilles tendon rupture $(15,16)$. A higher score on a scale of 0-100 indicates better functional outcome. The group minimal detectable change in the ATRS was reported to be 3.5 (17). Secondary outcomes included the duration of time it took to walk again normal- ly, and the incidence of re-rupture. All three variables were assessed using the survey.

\section{Determining NSAID Use}

NSAID use was determined using the survey (Figure 1). Patients were categorized as either NSAID users or non-NSAID users. Non-NSAID users may have used other pain medications or none at all.

\section{Exclusions}

In addition to excluding patients for uncertainty about pain medication use (Figure 1), patients were excluded if they were pregnant, used steroids, or used fluoroquinolones at any point during recovery (Figure 2 ). These exclusion criteria were selected because pregnancy, steroid use, and fluoroquinolone use are known to alter tendon characteristics (18-20). If patients were unsure if they took steroids or antibiotics, their prescription records were reviewed. If records

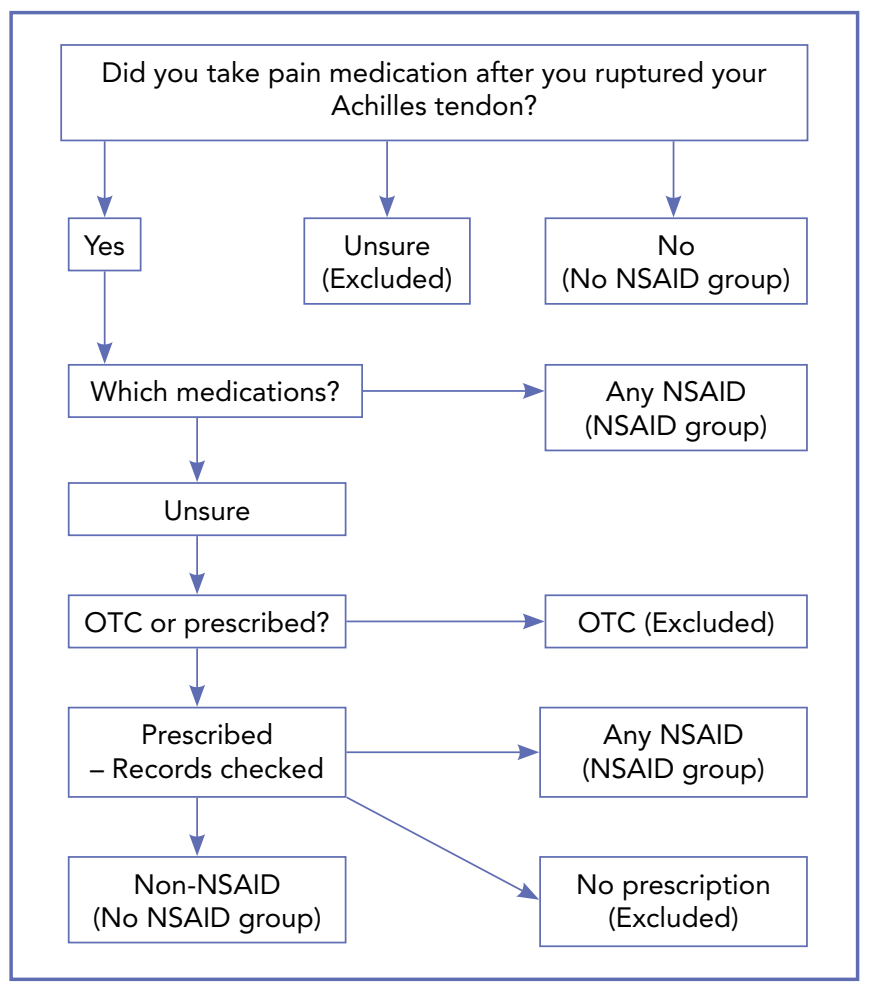

Figure 1. Diagram depicting how NSAID use was determined using the survey. Resulting groups are denoted in parentheses. Some patients endorsed taking pain medication, but were unsure of the specific type. If the unknown medication was over the counter (OTC), then they were excluded. If the unknown medication was prescribed, their prescription records were reviewed to determine which pain medications they had filled. 


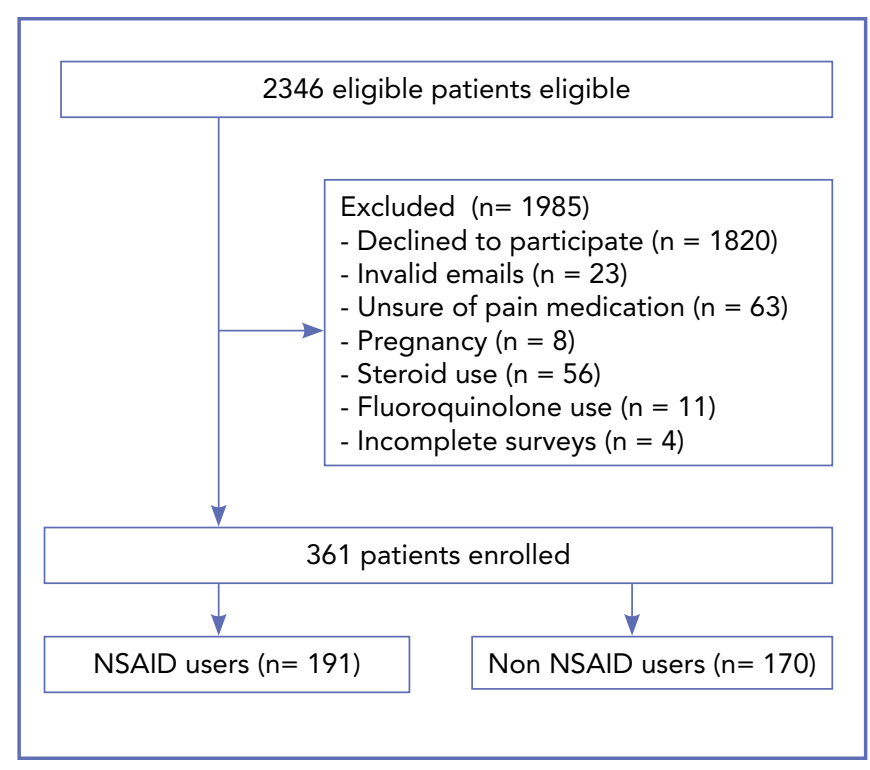

Figure 2. Diagram depicting number eligible, excluded, and enrolled in the study.

showed that these patients had filled a prescription for oral steroids or fluoroquinolones since their Achilles tendon rupture, they were excluded.

\section{Demographics}

Chart review was utilized to obtain demographic information such as age, sex, and operative versus non-operative repair.

\section{Statistical Analysis \\ ATRS}

The ATRS values were not normally distributed according to both the D'Agostino-Pearson normality test and the Shapiro Wilk normality test. We performed non-parametric Mann Whitney tests as well as parametric t-tests (as per the asymptomatic theory since our sample size is large), but the results and conclusions were not different. Thus, we only reported the results of nonparametric Mann-Whitney tests comparing the ATRS of NSAID users to non-NSAID users, the ATRS of females to that of males, and the ATRS of those who underwent operative versus non-operative repair. After appropriate diagnostics were confirmed (such as a normal distribution of the residuals), a multivariable linear regression model of the ATRS was performed with independent variables including age, NSAID use, sex, operative versus non-operative repair, and time from diagnosis to survey participation. For all statistical comparisons, the finding was considered statistically significant if $\mathrm{p}<0.05$. Values in parenthesis are medians.

\section{Time to Walk Normally}

A univariate cox proportional hazards model was used to quantify the association between NSAID use and the duration of time it took patients to walk again normally because 1) some respondents still did not walk normally at the time of the survey and 2) the time from diagnosis to survey was variable across subjects. The cumulative probability of a patient walking again normally after Achilles tendon rupture was computed across time. Each time point reflected either the time elapsed between tendon rupture and survey administration if the patient could not yet walk normally, or the time it took the patient to walk again normally, whichever came first.

\section{Incidence of Re-rupture}

Patients were asked whether or not they re-ruptured their Achilles tendon on the survey. The relationships between NSAID use, sex, and the incidence of Achilles tendon re-rupture were examined using Fisher's exact tests.

\section{RESULTS}

\section{Demographics}

Of the 2346 patients who were deemed eligible and sent email invitations to participate in the study, 1985 were excluded, leaving 361 patients in the study (Figure 2). Among survey responders, the median age was 47 (range = 18-96; interquartile range $[\mathrm{IQR}]=37-58$ ). Two-hundred and fifty responders were male. Two-hundred and nineteen had undergone non-operative repair of their Achilles tendon rupture. Fifty-three percent of responders $(\mathrm{n}=191)$ reported using NSAIDs. Surveys were completed a median of 24.3 months after Achilles tendon rupture (range $=4-51$ range; IQR $=12-33$ ).

Demographic information of NSAID users compared to non-NSAID users is displayed in Table I. Eleven patients (8 NSAID, 3 non-NSAID) entered an invalid identification number so age, sex, type of repair, and months from diagnosis to survey were only available for 350 patients. Seven patients did not provide ATRS data. Three patients did not provide re-rupture information. Thirteen patients did not respond to the walking again normally questions. The number of months from diagnosis to walking was computed from 286 patients because 62 patients responded that they still did not walk normally.

\section{ATRS}

Across all survey responders, the median ATRS was 73 $($ range $=10-100$ range; IQR $=49-90)$. ATRS data were missing from 7 patients due to incomplete responses, leav- 
Table I. Characteristics of NSAID users and Non-NSAID users

\begin{tabular}{llll}
\hline Variable & No NSAIDs $(\mathbf{n}=\mathbf{1 7 0})$ & NSAIDs $(\mathbf{n}=\mathbf{1 9 1})$ & p value \\
\hline Age & $48(18-83 ; 33-60)$ & $46(22-96 ; 37-56)$ & 0.65 \\
\hline Male & $117(72 \%)$ & $133(71 \%)$ & 0.813 \\
\hline Female & $45(28 \%)$ & $55(29 \%)$ & 0.912 \\
\hline Non-Operative & $102(63 \%)$ & $117(62 \%)$ & 0.005 \\
\hline Operative & $60(37 \%)$ & $71(38 \%)$ & 0.399 \\
\hline ATRS ** & $77(11-100 ; 56-93)$ & $69(10-100 ; 45-87)$ & \\
\hline No Re-rupture & $160(95 \%)$ & $174(92 \%)$ & 0.987 \\
\hline Re-rupture & $9(5 \%)$ & $15(8 \%)$ & 0.326 \\
\hline Mos from dx to survey & $22.3(4-51 ; 12-32)$ & $24.8(4-51 ; 13-33)$ & \\
\hline Wot Walking Normally & $25(15.3 \%)$ & $37(20 \%)$ & $148(80 \%)$ \\
\hline
\end{tabular}

Continuous variables are summarized by median (range; interquartile range [IQR]). Discrete variables are summarized as number per group (\% of group). Ranges and IQR were rounded to the nearest whole number. **indicates $\mathrm{p}<0.05$ for Mann Whitney tests when comparing the NSAID to no NSAID groups. Months is abbreviated as Mos.

ing 354 patients for this analysis. NSAID users reported a significantly lower ATRS (median $=69 ; \mathrm{IQR}=45-87$ ) than non-NSAID users (median 77; IQR $=56-93 ; \mathrm{p}=$ 0.005). Figure 3 illustrates distributions of the ATRS across NSAID users and non-NSAID users. The lower ATRS ranges are characterized predominantly by NSAID users while the higher ATRS ranges are characterized primarily by non-NSAID users.

There was a significant correlation between the ATRS and the months that elapsed between the time of diagnosis and survey completion $(\mathrm{p}<0.0001)$. Thus, the number of months between diagnosis and survey participation was included as an independent variable in the multivariable linear regression model. After controlling for age, sex, operative versus non-operative repair, and time from diagnosis to survey completion, NSAID use was still associated with a significantly lower ATRS $(\mathrm{p}=0.003)$.

Incidentally, females (median $=61 ; \mathrm{IQR}=40.8-83$ ) reported a significantly lower ATRS than males (median $=77$; IQR = 55-93; $\mathrm{p}<0.001)$. Even after controlling for age, NSAID use, operative versus non-operative repair, and time from diagnosis to survey completion, female sex was still associated with a significantly lower ATRS $(\mathrm{p}<0.001)$. There was no difference in the percent of females $(30 \%)$ and males $(40 \%)$ choosing to undergo surgery $(\mathrm{p}=0.09)$. Males and females exhibited no significant difference in age $(\mathrm{p}=0.44)$, or months from diagnosis to survey $(\mathrm{p}=0.45)$.

Patients who reported undergoing operative repair reported a higher ATRS (median $=77 ; \mathrm{IQR}=54-93$ ) than those who underwent non-operative repair (median $=70$; IQR
$=46-88 ; \mathrm{p}=0.03)$. However, when controlling for age, NSAID use, time from diagnosis to survey completion and sex, operative repair was no longer associated with a higher ATRS ( $p=0.093)$. Of note, those who underwent operative repair were significantly younger (median $=43$; IQR =33-50) than those who underwent non-operative repair (median $=49 ; \mathrm{IQR}=39-61 ; \mathrm{p}<0.0001)$.

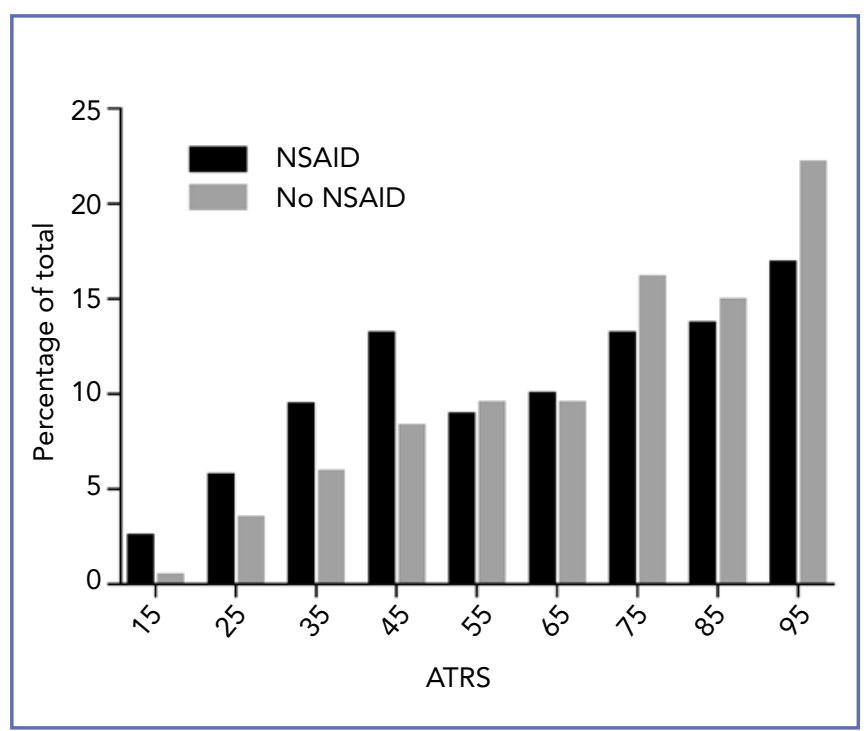

Figure 3. The percent of NSAID users and non-NSAID users who reported an ATRS in each of the listed ranges. Each bin spans 10 ATRS points. The middle of each 10-point range is labeled on the $\mathrm{x}$-axis. 


\section{Time to Walk Again Normally}

At the time of the study, 287 patients reported that they were walking normally, while 61 stated that they were still not walking normally. Thirteen patients declined to answer this question. Of the 287 patients who reported walking again normally at the time of the survey, the median time to walk was 6 months (range $=0.5-36 ; \mathrm{IQR}=4-8$ ). NSAID use was not significantly associated with the amount of time it took patients to walk again normally after an Achilles tendon rupture using the cox proportional hazards model $(\mathrm{p}=0.094 ;$ Figure 4A).

Because female sex was noted to be associated with a lower ATRS, the relationship between sex and the secondary outcomes of time to walk again normally and re-rupture rate were also examined. A cox proportional hazards model demonstrated that female sex $(\mathrm{p}<0.001$; Figure 4B) was associated with a significantly longer time to walk again normally after Achilles tendon rupture.

A

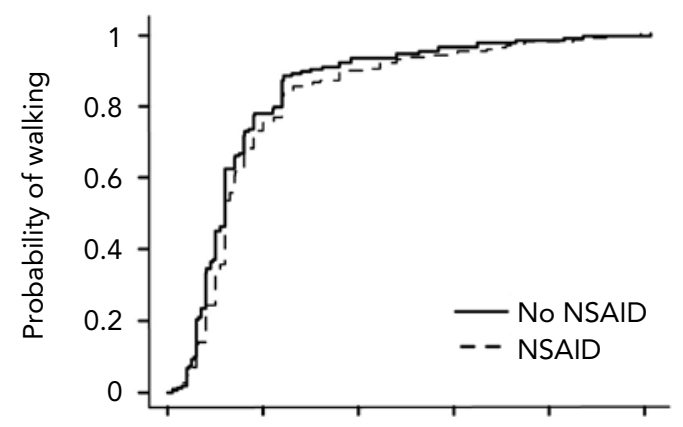

B

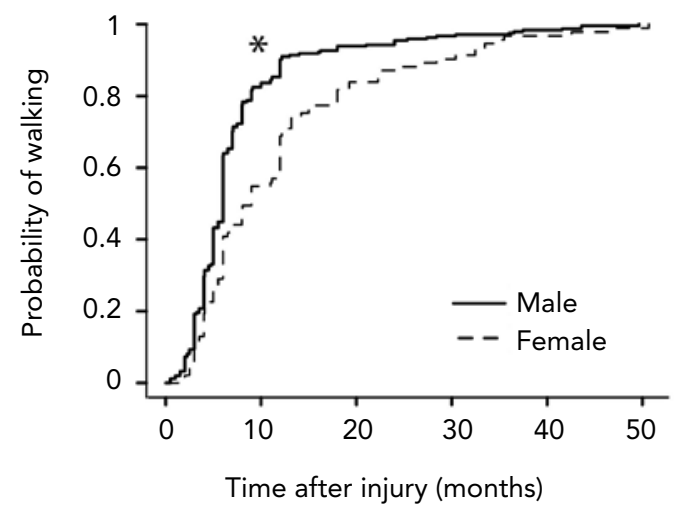

Figure 4. Cox proportional hazards models showing the relationship between the probability of time to walk again normally after injury and A) NSAID use as well as B) sex. ${ }^{*}$ denotes $p$ $<0.05$.

\section{Incidence of Achilles Tendon Re-rupture}

Twenty-four patients $(6.7 \%)$ reported having an Achilles tendon re-rupture. Three patients declined to answer this question. Fifteen patients $(7.9 \%)$ in the NSAID group reported re-rupture, while nine $(5.3 \%)$ of the non-NSAID users reported re-rupture $(\mathrm{p}=0.399)$. Eight females $(8.2 \%)$ reported a re-rupture, while 14 males $(5.6 \%)$ reported a re-rupture $(\mathrm{p}=0.46)$. Eight patients $(6.15 \%)$ who underwent surgical repair reported a re-rupture, whereas fourteen patients $(6.45 \%)$ who underwent non-operative treatment reported re-rupture $(\mathrm{p}>0.99)$.

\section{DISCUSSION}

This is the first study to examine the association between NSAID use and functional recovery in humans after Achilles tendon rupture. NSAID users reported a significantly lower ATRS as compared to non-NSAID users, suggesting worse functional outcomes. After controlling for variables such as sex, age, operative versus non-operative approach, and time from diagnosis to survey completion, NSAID use was still associated with a lower ATRS. NSAID use was not associated with a longer time to walk again normally or an increased incidence of re-rupture. However, the time to walk again normally was subject to recall bias and it is not an externally validated measure of functional recovery after rupture. A recent meta-analysis reported average overall re-rupture rates to be $5 \%(21)$, which is similar to the overall rate we found here of $6.7 \%$. Given the rarity of re-rupture, this study was likely underpowered to detect a difference in re-rupture rate between the groups.

An interesting association between female sex and a lower ATRS after Achilles tendon rupture was also discovered. This prompted the investigation of the relationship between female sex and other secondary measures of recovery after Achilles tendon rupture. Indeed, female sex was associated with a longer time to walk again normally.

Initially, those who had non-operative repair seemed to have a lower ATRS than those who underwent operative repair. However, after controlling for age, NSAID use, sex, and months from diagnosis to survey, this association was no longer present.

Due to the survey-based nature of this study, it is subject to recall bias. Patients may simply have not remembered whether or not they took NSAIDs, especially if they ruptured their Achilles tendon years ago. However, subjects were allowed to answer, "I don't know" and were later excluded if they were unsure. Prescription records were also reviewed to verify which prescriptions were filled if a patient stated that they were unsure of which prescribed medications they took (Figure 1). Still, patients may have taken an NSAID over 
the counter without remembering, which may have influenced results. While patients were not blinded to whether or not they took NSAIDs, it is unclear in which direction this would have biased patients as they were not informed beforehand of the hypothesis.

As this was a retrospective multi-center study involving patients with different providers, the post-operative and non-operative treatment protocols were not standardized across patients. This may have introduced additional bias, as there is no consensus on the optimal rehabilitation proto$\operatorname{col}(13)$.

Due to the follow-up time point of this study, patients who ruptured their tendon in 2014 had more time to recover than those who ruptured their tendon in 2018. Indeed, there was a correlation between the time from diagnosis to survey and the ATRS. To account for this, we included the time from diagnosis to survey completion as an independent factor in the multivariate regression analysis. After controlling for the time from diagnosis to survey, NSAIDs were still associated with a lower ATRS score. In the time to walk analysis, we employed a cox proportional hazards model to help account for the variability in time from diagnosis to survey as well.

Patients were not randomized to their treatment groups, which could have introduced selection bias. While Table I shows that the two groups were demographically similar, it is possible that there were unmeasured differences that influenced outcomes. For example, there may have been differences in the use of supplements, vitamins, or nutraceuticals between groups. Such substances may have anti-inflammatory properties themselves, or could exert synergistic or antagonistic effects on NSAIDs.

While there is a wide range of age groups included in this study, the age of NSAID users was not different from non-NSAID users, making it unlikely that having such a wide age range influenced the relationship between NSAID use and ATRS. Furthermore, NSAID use was still associated with a lower ATRS even after controlling for age.

Patients' injuries were not confirmed by uniform imaging as this was a retrospective study and patients were diagnosed clinically, by ultrasound, or with MRI. While this may have reduced accuracy in diagnosis, the ICD-9 and ICD-10 codes were used to identify eligible patients with initial encounters for Achilles tendon ruptures.

Importantly, only $15 \%$ of eligible patients were included in the final results. While 503 patients started the survey, only 361 completed the survey without exclusions. It is unclear how accurately this small proportion represents the entire population. The timing and dosage of NSAID administration may also be important in how NSAIDs influence recovery $(11,12)$ and was not evaluated here, representing another potential limitation.
It is possible that taking any pain medication after Achilles tendon rupture may influence healing. In this study, non-NSAID users included both patients who may have used other pain medications and those who used no pain medications at all. Further studies are necessary to tease apart how pain medications versus anti-inflammatory medications affect recovery.

Currently, the effect of NSAIDs on recovery after Achilles tendon rupture remains unknown. NSAIDs have been theorized to have deleterious effects on tendon healing $(5,6)$. Conversely, there is evidence that NSAIDs increase collagen synthesis (5), which could facilitate healing. Rodent studies evaluating the effects of NSAIDs on healing after transection of the Achilles tendon are conflicting $(7,10)$. These studies have limited applicability due to the lack of human subjects, the artificial surgical nature of injury, and the very early time points of assessment following injury. This is the first study to examine the relationship between NSAID use and recovery after Achilles tendon rupture in humans.

The only study to date looking at the effects of NSAIDs on Achilles tendon injury in humans was performed on patients with Achilles tendinopathy rather than rupture. Seventy patients were given perixocam or placebo and re-assessed twenty-eight days later (22). The authors reported no difference in recovery with NSAID use; however, the sample size was small, the drug selection was atypical, the follow-up time point was early, and the study did not involve patients with Achilles tendon rupture.

Consistent with our work, ketorolac administration following anterior cruciate ligament (ACL) reconstruction was associated with worse outcomes in one study (23). Conversely, another study showed NSAID use was associated with equivalent Visual Analog Scale pain scores and examination one year after operative ACL repair (24).

In agreement with our data, prior work has reported that females with Achilles tendon rupture suffer worse outcomes than males (25). The etiology of this sex-specific difference in healing is currently unclear. It is unlikely that this sex-specific difference in recovery influenced the association between NSAID use and the ATRS that we observed. First, there were similar proportions of females in the NSAID and non-NSAID group (Table I). Furthermore, a regression analysis demonstrated an association between NSAID use and a lower ATRS even after controlling for sex as an independent variable.

In support of our finding that operative versus non-operative repair did not affect the ATRS after Achilles tendon rupture (after controlling for variables such as age), a meta-analysis found no difference in the ATRS after operative and non-operative treatment of Achilles tendon ruptures (26). However, one randomized controlled trial reported that 
surgical repair of the Achilles tendon was associated with better physical functioning (27). Any potential association between operative repair and improved outcomes did not influence the association between NSAID use and ATRS that we observed. There were similar numbers of operative and non-operative subjects in the NSAID and no NSAID group (Table I). Additionally, the association between NSAID use and a lower ATRS was still present after controlling for treatment approach in the regression analysis.

\section{CONCLUSIONS}

NSAID use after Achilles tendon rupture was associated with a lower ATRS. While this study is insufficient to demonstrate a causal relationship between NSAID use and

\section{REFERENCES}

1. Zhang X, Schwarz EM, Young DA, Puzas JE, Rosier RN, O'Keefe RJ. Cyclooxygenase-2 regulates mesenchymal cell differentiation into the osteoblast lineage and is critically involved in bone repair. The Journal of clinical investigation. 2002 Jun;109(11):1405-15. PubMed PMID: 12045254. Pubmed Central PMCID: 151001.

2. Simon AM, Manigrasso MB, O'Connor JP. Cyclo-oxygenase 2 function is essential for bone fracture healing. Journal of bone and mineral research : the official journal of the American Society for Bone and Mineral Research. 2002 Jun;17(6):963-76. PubMed PMID: 12054171.

3. Dodwell ER, Latorre JG, Parisini E, et al. NSAID exposure and risk of nonunion: a meta-analysis of case-control and cohort studies. Calcified tissue international. 2010 Sep;87(3):193-202. PubMed PMID: 20552333.

4. Bhattacharyya T, Levin R, Vrahas MS, Solomon DH. Nonsteroidal antiinflammatory drugs and nonunion of humeral shaft fractures. Arthritis and rheumatism. 2005 Jun 15;53(3):364-7. PubMed PMID: 15934108.

5. Tsai WC, Hsu CC, Chou SW, Chung CY, Chen J, Pang JH. Effects of celecoxib on migration, proliferation and collagen expression of tendon cells. Connective tissue research. 2007;48(1):46-51. PubMed PMID: 17364667.

6. Tsai WC, Hsu CC, Chang HN, Lin YC, Lin MS, Pang JH. Ibuprofen upregulates expressions of matrix metalloproteinase-1, $-8,-9$, and -13 without affecting expressions of types I and III collagen in tendon cells. Journal of orthopaedic research : official publication of the Orthopaedic Research Society. 2010 Apr;28(4):487-91. PubMed PMID: 19847888.

7. Hammerman M, Blomgran P, Ramstedt S, Aspenberg P. COX-2 inhibition impairs mechanical stimulation of early tendon healing in rats by reducing the response to microdamage. Journal of applied physiology. 2015 Sep 01;119(5):534-40. PubMed PMID: 26159755.

8. Dimmen S, Engebretsen L, Nordsletten L, Madsen JE. Negative effects of parecoxib and indomethacin on tendon healing: an experimental study in rats. Knee surgery, sports trau- worse recovery after Achilles tendon rupture, it highlights the importance of conducting future randomized, blinded studies to further delineate the relationship between NSAID use and recovery after Achilles tendon rupture. The effects of NSAID timing and dosage on recovery and the molecular pathways whereby NSAIDs impact tendon healing will be an important consideration. Additionally, females reported significantly worse recovery after Achilles tendon rupture than males. Future studies are needed to determine the etiology of this sex-specific disparity so that recovery in females may be optimized.

\section{Conflict of interest}

The authors report no conflicts of interest or disclosures.

matology, arthroscopy : official journal of the ESSKA. 2009 Jul;17(7):835-9. PubMed PMID: 19296084.

9. Ferry ST, Dahners LE, Afshari HM, Weinhold PS. The effects of common anti-inflammatory drugs on the healing rat patellar tendon. The American journal of sports medicine. 2007 Aug;35(8):1326-33. PubMed PMID: 17452512.

10. Forslund C, Bylander B, Aspenberg P. Indomethacin and celecoxib improve tendon healing in rats. Acta orthopaedica Scandinavica. 2003 Aug;74(4):465-9. PubMed PMID: 14521300.

11. Connizzo BK, Yannascoli SM, Tucker JJ, et al. The detrimental effects of systemic Ibuprofen delivery on tendon healing are time-dependent. Clinical orthopaedics and related research. 2014 Aug;472(8):2433-9. PubMed PMID: 23982408. Pubmed Central PMCID: 4079885.

12. Virchenko O, Skoglund B, Aspenberg P. Parecoxib impairs early tendon repair but improves later remodeling. The American journal of sports medicine. 2004 Oct-Nov;32(7):1743-7. PubMed PMID: 15494342.

13. Oliva FR, Clelia \& Giai Via, A \& Baldassarri, Matteo \& G, Bernardi \& Biz, Carlo \& Bossa, M \& Buda, Roberto \& Buonocore, D \& Chianca, Vito \& A, Collina \& De Carli, A \& De Luna, A.V. \& Di Lanno, I \& di lorenzo, Luigi \& Di Pietto, Francesco \& Dossena, M \& Fantoni, Ilaria \& Farsetti, P \& Maffulli, Nicola. I.S.Mu.L.T. Achilles tendon ruptures guidelines. Muscles Ligaments Tendons Journal. 2018;8(3):310-63.

14. Padulo J, Oliva F, Frizziero A, Maffulli N. Muscles, Ligaments and Tendons Journal - Basic principles and recommendations in clinical and field Science Research: 2016 Update. Muscles, ligaments and tendons journal. 2016 Jan-Mar;6(1):1-5. PubMed PMID: 27331026. Pubmed Central PMCID: 4915447.

15. Kearney RS, Achten J, Lamb SE, Parsons N, Costa ML. The Achilles tendon total rupture score: a study of responsiveness, internal consistency and convergent validity on patients with acute Achilles tendon ruptures. Health and quality of life outcomes. 2012 Feb 29;10:24. PubMed PMID: 22376047. Pubmed Central PMCID: 3305449.

16. Nilsson-Helander K, Thomee R, Silbernagel KG, et al. The Achilles tendon Total Rupture Score (ATRS): development 
and validation. The American journal of sports medicine. 2007 Mar;35(3):421-6. PubMed PMID: 17158277.

17. Opdam KTM, Zwiers R, Wiegerinck JI, et al. Reliability and validation of the Dutch Achilles tendon Total Rupture Score. Knee surgery, sports traumatology, arthroscopy : official journal of the ESSKA. 2018 Mar;26(3):862-8. PubMed PMID: 27417101. Pubmed Central PMCID: 5847201.

18. Kirchgesner T, Larbi A, Omoumi P, et al. Drug-induced tendinopathy: from physiology to clinical applications. Joint, bone, spine : revue du rhumatisme. 2014 Dec;81(6):485-92. PubMed PMID: 24962977.

19. Knobloch K. Drug-Induced Tendon Disorders. Advances in experimental medicine and biology. 2016;920:229-38. PubMed PMID: 27535265.

20. Schauberger CW, Rooney BL, Goldsmith L, Shenton D, Silva PD, Schaper A. Peripheral joint laxity increases in pregnancy but does not correlate with serum relaxin levels. American journal of obstetrics and gynecology. 1996 Feb;174(2):667-71. PubMed PMID: 8623804.

21. Wu Y, Mu Y, Yin L, Wang Z, Liu W, Wan H. Complications in the Management of Acute Achilles Tendon Rupture: A Systematic Review and Network Meta-analysis of 2060 Patients. The American journal of sports medicine. 2019 Feb 19:363546518824601. PubMed PMID: 30781966.

22. Astrom M, Westlin N. No effect of piroxicam on achilles tendinopathy. A randomized study of 70 patients. Acta orthopaedica Scandinavica. 1992 Dec;63(6):631-4. PubMed PMID: 1471511.
23. Mehta VM, Young EP, Paxton EW, Fithian DC. The effect of ketorolac on anteroposterior knee laxity after anterior cruciate ligament reconstruction. Orthopedics. 2008 Jun;31(6):538-40. PubMed PMID: 18661874.

24. Ge H, Liu C, Shrestha A, Wu P, Cheng B. Do Nonsteroidal Anti-Inflammatory Drugs Affect Tissue Healing After Arthroscopic Anterior Cruciate Ligament Reconstruction? Medical science monitor : international medical journal of experimental and clinical research. 2018 Aug 30;24:603843. PubMed PMID: 30160247. Pubmed Central PMCID: 6128182 .

25. Silbernagel KG, Brorsson A, Olsson N, Eriksson BI, Karlsson J, Nilsson-Helander K. Sex Differences in Outcome After an Acute Achilles Tendon Rupture. Orthopaedic journal of sports medicine. 2015 Jun;3(6):2325967115586768. PubMed PMID: 26665094. Pubmed Central PMCID: 4622365.

26. Deng S, Sun Z, Zhang C, Chen G, Li J. Surgical Treatment Versus Conservative Management for Acute Achilles Tendon Rupture: A Systematic Review and Meta-Analysis of Randomized Controlled Trials. The Journal of foot and ankle surgery : official publication of the American College of Foot and Ankle Surgeons. 2017 Nov - Dec;56(6):1236-43. PubMed PMID: 29079238.

27. Lantto I, Heikkinen J, Flinkkila T, et al. A Prospective Randomized Trial Comparing Surgical and Nonsurgical Treatments of Acute Achilles Tendon Ruptures. The American journal of sports medicine. 2016 Sep;44(9):2406-14. PubMed PMID: 27307495. 